\title{
Estrategia de Operación de Subestaciones en la Corporación Nacional de Electricidad Empresa Pública Unidad de Negocio Milagro: Caso Subestación Marcelino Maridueña
}

\author{
Ing. Abel Rodolfo Carrasco Andrade, \\ Universidad Estatal de Milagro, Ecuador \\ Félix Enrique Villegas Yagual, PhD \\ Docente Facultad de Ciencias Administrativas y Comerciales \\ Universidad Estatal de Milagro, Ecuador
}

Doi: 10.19044/esj.2019.v15n6p456 URL:http://dx.doi.org/10.19044/esj.2019.v15n6p456

\section{Resumen}

Un requisito indispensable para solucionar los problemas de confiabilidad del sistema eléctrico de potencia es tener conocimiento acerca de los equipos de la subestación, el monitoreo de variables eléctricas y la lectura y contratación de parámetros eléctricos en equipos de potencia.Por tal motivo surge la necesidad de implementar un sistema de potencia idóneo y confiable en cargabilidad, niveles de tensión y calidad de servicio. En el presente estudio a partir de la metodología de investigación de análisis de campo, se lleva a cabo el monitoreo y diseño de implementación de una estrategia de operación satisfactoria que disminuya las distorsiones de onda de voltaje producidas por transitorios eléctricos en el momento de interconectar y transmitir la energía de la subestación Marcelino Maridueña desde la línea de Subtransmision Milagro 3 a Milagro 4, esto con el objetivo de optimizar recursos y minimizar tiempos de respuesta para maniobras de equipos en Alta y Media Tensión ante cualquier contingencia de desconexión. Este proyecto involucra de igual manera un análisis de evaluación de proyectos y tasas de rentabilidad económica, obteniendo indicadores económicos satisfactorios.

Palabras claves: Sistema eléctrico, Subestación, Equipos de potencia, Estrategia de operación, Indicadores económicos 


\title{
Strategy of Operation of Sub-Stations in the National Corporation of Electricity Public Company Unit of Business Milagro: A Case of Marcelino Maridueña Sub-Station
}

\author{
Ing. Abel Rodolfo Carrasco Andrade, \\ Universidad Estatal de Milagro, Ecuador \\ Félix Enrique Villegas Yagual, PhD \\ Docente Facultad de Ciencias Administrativas y Comerciales \\ Universidad Estatal de Milagro, Ecuador
}

\begin{abstract}
An indispensable requirement to solve reliability problems of the electrical power system is to have knowledge about the equipment of the substation, the monitoring of electrical variables, and the reading and contracting of electrical parameters in power equipment. For this reason, there arises a need to implement an ideal and reliable power system in terms of chargeability, voltage levels, and quality of service. In the present study, based on the field analysis research methodology, the monitoring and design of implementation of a satisfactory operation strategy is carried out to reduce the voltage wave distortions produced by electrical transients. This happens when the energy of the Marcelino Maridueña substation is interconnected and transmitted from the subtransmission line Milagro 3 to Milagro 4. The aim is to optimize resources and minimize response times for equipment maneuvers in High and Medium Voltage before any disconnection contingency. This paper, however, focuses on analyzing the project evaluation and economic rates of return, as well as obtaining satisfactory economic indicators.
\end{abstract}

Keywords: Electrical system, Substation, Power equipment, Operation strategy, Economic indicators

\section{Introduction}

A raíz de la incorporación del Estado Ecuatoriano al desarrollo de las actividades de gestión pública basadas en Tecnologías de la Información y Comunicación (TICs) orientadas a mejorar la provisión de servicios, acercamientos y las relaciones de la Administración del sector público, la 
Corporación Nacional de Electricidad (CNEL EP) implementó un Sistema de Atención al Reclamo (SAR), plataforma informática que permite establecer un vínculo directo con el usuario, donde el abonado podrá reportar anomalías del sector energético tipo residencial, comercial e Industrial. Con la herramienta tecnologica SAR, la Corporación Nacional de Electricidad tiene la obligación de tener un Sistema de Distribución de Energía con un alto índice de confiablidad y calidad de servicio para sus abonados.

Bajo este contexto, se considera al Sistema de Atención al Reclamo como una herramienta del Gobierno Electrónico, la cual permite establecer indicadores de satisfacción por el servicio eléctrico en empresas distribuidoras de energia eléctrica, teniendo prinicipal transcendecencia en informes de rendición de cuentas donde el Estado Ecuatoriano informa a sus habitantes. Por los antecedentes anteriormente presentados, CNEL EP apuesta por la automatización de sus principales elementos de distribución como son las subestaciones y líneas de Subtransmisión, con el único objetivo de optimizar recursos y minimizar tiempos de respuesta para maniobras de equipos en Alta y Media Tensión ante cualquier contingencia de desconexión.Esto se lleva a cabo enfocándose en los Objetivos Nacionales de Desarrollo, más específicamenteapegándose a los objetivos 4,5, para así garantizar la productividad, soberanía y servicio social.

Objetivo 4: Consolidar la sostenibilidad del sistema económico social y solidario, y afianzar la dolarización (Secretaría Nacional de Planificación \& Desarrollo, 2017). Siendo CNEL EP UNMLG una empresa considerada como estratégica que permitirá transformar la economía, fortaleciendo la matriz productiva y económica del canton Marcelino Maridueña.

Objetivo 5: Impulsar la productividad competitividad para el crecimiento económico sostenible de manera redistributiva y solidaria (Secretaría Nacional de Planificación \& Desarrollo, 2017). CNEL EP UNMLG creará fuentes de trabajo en el campo tecnológico tanto público como privado; ademas brindará un sistema eléctrico confiable, óptimo para la innovación empresarial creando oportunidades para el una mejor calidad de vida.

Siendo la Corporación Nacional de Electricidad una empresa estratégica destinada al desarrollo sustentable del país, es importante señalar que las empresas deben cumplir con una lógica de procesos en la operación del sistema de distribución y Subtransmisión, automatización que se lleva a cabo implementando tecnologías informáticas para la interacción entre los departamentos de operación y mantenimiento de CNEL EP UN Milagro y la distribuidora de energía eléctrica.

En la actualidad se debe implementar un modelo de gestión de procesos para coadyuvar a las diferentes áreas de una empresa distribuidora como es el caso de CNEL EP UN MLG. Este modelo de gestión deberá 
unificar el proceso de operación y mantenimiento del sistema eléctrico aplicando un mantenimiento predictivo, preventivo y correctivo de sistemas eléctricos. En tal sentido, se justifica esta investigacion por la necesidad de aumentar la confiabilidad del sistema eléctrico de potencia, disminuyendo las fallas generadas por maniobras en equipos realizadas manualmente, bajo condiciones de riesgo mecánico y eléctrico. Para ello se contribuirá con un diseño estratégico de operación en maniobras en equipos de potencia y unaplanificación de estructura automatizada de subestaciones, incluyendo un sistema integral en equipos de potencia en media y alta tensión en tiempo real.

Se plantea entonces como objetivo de investigación dar a conocer de forma efectiva el impacto de la implementación de una estrategia de operación de subestaciones y líneas de subtransmisión para el caso de la subestación Marcelino Maridueña, cuyo objeto es mejorar la calidad y continuidad del servicio eléctrico a nivel industrial, residencial y comercial en el cantón Marcelino Maridueña. Para alcanzar dicho objetivo se han planteado los siguientes puntos:

- Analizar, estandarizar y automatizar los equipos de la subestación.

- Diseñar una estrategia de operación de subestaciones y líneas de Subtransmisión.

- Instalar mediante protección automática y seccionamiento en el sistema eléctrico una plataforma georreferenciada.

- Implementar el mantenimiento del sistema eléctrico.

\section{Hipótesis}

$\mathrm{H}_{1}$ 'Diseñar una estrategia de operación de líneas de subtransmisión mediante la automatización de equipos lo que permitirá disminuir el tiempo de desconexión, ocasionando así un impacto positivo en la variable "confiabilidad" del sistema eléctrico de potencia de la subestación.

$\mathrm{H}_{2}$ : El sistema de monitoreo SCADA obtendrá mediciones en tiempo real en los equipos de la subestación y líneas de subtransmisión, las cuales permitirán tener mayor selectividad en los equipos. El operario, mediante la automatización de equipos, restablecerá el flujo eléctrico con mayor rapidéz, afectando positivamente en la disminución de índices en parámetros FMIK y TTIK reportados al ARCONEL.

$\mathrm{H}_{3}$ : Con la implementación de un sistema de potencia eliminador de transitorios eléctricos descartaremos las desconexiones de clientes de tipo industrial del cantón Marcelino Maridueña, lo que conlleva a que no se desconecten del suministro eléctrico que brinda CNEL EP UN MLG y autogeneren, ocasionando pérdidas económicas para la empresa distribuidora. 


\section{Desarrollo}

Diseñar y proponer un modelo de gestión por procesos, el cual permita administrar sus recursos, incrementar su productividad, conseguir la satisfacción de sus clientes, mejorar sus procesos internos y controlar de manera adecuada sus resultados permitiendo tomar decisiones a tiempo con medidas preventivas y correctivas (Wendy Paola Perugachi Flores, 2018). En CNEL EP UN MLG es necesario un modelo de gestión que ayudará administrar de mejor manera los recursos de forma efectiva y confiable con el objetivo de maximizar la productividad, minimizar gastos operativos y no operativos y sereficaces en las tomas de decisiones, todo esto en busca del beneficio social y la interacción entre los usuarios, el Centro de Operaciones, el Departamento de Subestaciones y el CENACE.

Esta necesidad junto con la viabilidad y disponibilidad de los servicios de comunicación a distancia se juntan para crear una alternativa de solución a las necesidades de monitoreo de los equipos o maquinarias mediante módulos del tipo Maestro-Esclavo.

El esclavo va conectado directamente a las máquinas - emisor, mientras que el Maestro - receptor se encuentra en un tablero; la comunicación se realiza mediante un bus de datos RS-485. (Mendoza Segura, 2017). E1 monitoreo de las señales eléctricas de las subestaciones eléctricas que es el objeto de análisis del presente artículo, se basa en la implementación de un sistema de potencia, con medición de voltaje y corriente con fasores de los mismos, que se deriva de la potencia aparente, reactiva y activa. La interconexión de señales medibles entre el tablero del equipo a ser monitoreado y el Reuter de control se realiza mediante un bus de datos con puerto de comunicación RS-485, permitiendo así presentar un reporte histórico de equipos.

Se desglosa la automatización de subestaciones en una empresa ubicándolas geográficamente y enlistándolas, diferenciando la automatización en términos de equipos y comunicaciones y evaluando estas características de automatización. (Hurtado Avélla \& Santiago Andrés, 2016) La interacción, integración de parámetros eléctricos a un sistema de navegación y control se realiza mediante niveles de comunicación y enlace de equipos de control, protección y adquisición de datos. Estos niveles deben ser interpretados en base a la funcionabilidad y grado de seguimiento de equipos, con respecto a la versatilidad del proyecto.

Interrupciones por descargas atmosféricas ocasionadas por diversos factores como; la densidad de impactos de rayo, la altura de la línea aérea del cable de guarda y las diferentes distancias mínimas que caracterizan a una línea de media tensión (Quispe Flores \& Juvenal Antonio, 2018). En pocas palabras la confiabilidad del sistema eléctrico en CNEL EP UN MLG es medible en referencia a la capacidad de suministrar energía, garantizando la 
continuidad y calidad de servicio; la primera es relacionada a distorsiones a la forma de ondas de voltaje, corriente y potencia, mientras que la segunda se refiere a la frecuencia de desconexión, medible en períodos de afectación de continuidad del fluido eléctrico.

\section{Justificación}

Con el objetivo de justificar el análisis de la subestación Marcelino Maridueña se ha realizado un estudio de campo, recopilando información de cargabilidad de la subestación Reductora, así como también de la línea de Subtransmisión Milagro 3 y Milagro 4, suministro de energía e índices de desconexión.

En caso contrario si se trata del usuario final, éste deberá conocer la importancia de su negocio y lo catastrófico que resultaría la pérdida de suministro eléctrico a sus cargas críticas, por lo tanto comprará lo que él considere que resuelve mejor su problema a largo plazo y no necesariamente la propuesta con el "menor precio" (Gutierrez Lascurain Isabela, 2012). A pesar de que la subestación Marcelino Maridueña esla única distribuidora de energía eléctrica dentro del área de cobertura del cantón, las empresas industriales a $69 \mathrm{kV}$ poseen la libertad de invertir en sus empresas con el objetivo de generar electricidad y/o depender del suministro que brinda CNEL EP UN MLG. Como afirma Gutiérrez Kascurain, 2012,“ el usuario final deberá conocer la importancia de su negocio y lo catastrófica que resultaría la pérdida de suministro eléctrico a sus cargas críticas, por lo tanto comprará lo que él considere que resuelve mejor su problema a largo plazo y no necesariamente la propuesta con el "menor precio". Por los motivos expuestos se manifiestala necesidad de dimensionar de manera efectiva la cargabilidad, calidad de servicio en niveles óptimos de voltaje pero sobre todo poseer una confiabilidad en su sistema eléctrico de potencia, con el objetivo de que las industrias del cantón Marcelino Maridueña dependan únicamente del suminstro que brinda la CNEL EP.

La pérdida de suministro de energía eléctrica por interrupción o por transferencia de energía entre las líneas de Subtransmision Milagro 3 y Milagro 4ocasiona una pérdida en dinero para la empresa distribuidora, ocasiona malestares a las empresas industriales antes mencionadas, hace que aumente la no confiabilidad del sistema eléctrico, y por lo tanto esto conlleva también a una disminución de clientes. El tiempo en que la subestación deja de suministrar energía a las empresas industriales representa un valor económico de \$33,048.00al año.

Por esta razón nos basamos en los precios que establece el gobierno mediante su organismo de control, lo que indica que su costo de energía renovable es de \$0.09 dólares (Agencia de Regulacion \& Control de Electricidad, 2018). 
Cliente industrial en alta tensión.

Energía entregada: $10.20 \mathrm{MW}$

Frecuencia de interrupción: $2 /$ mes $=36$

Valor generadora renovable - quema de Biomasa: $\$ 33,048.00$

Tiempo de interrupción: $15 \mathrm{~min}=0.25 \%$ / hora

Principal objetivo de la política energética, el cual consiste en garantizar de manera eficiente el suministro y confiablidad en la prestación del servicio (Garcia Jhon - Rendon, 2018), La confiabilidad del Sistema Eléctrico de Potencia es el principal problema de una empresa distribuidora de energía eléctrica tipo pública ya que representa un peligro eminente para el factor humano tanto en la transferencia como para los equipos de las empresas industriales.

CNEL EP UN MLG obtiene una pérdida de suministro de energía en el momento de la desconexión. El Ingenio San Carlos durante la epoca de zafra genera electricidad por medio de la quema del bagazo proveniente de la caña de azúcar - biomasa, energía que no es $100 \%$ confiable por no ser constante en tiempo ni en cantidad de caña recolectada. Esta energía generada por biomasa esposteriormente suministrada a la red del Sistema Nacional Interconectado a través de la línea de Subtransmisión Milagro 3, la cual no es suficiente para un período superior a 1 hora representado en $\$ 33,048.00$ debido a que las autogeneradoras no tienen capacidad de generar electricidad a frecuencia constante. Por tal motivo, se apuesta a la creación de una estrategia de operación de subestaciones la cual conlleva al monitoreo, maniobras de equipos eléctricos en lineas de subtransmisión durante el período en que la subestación queda sin energía el cual es de 15 minutos.

Al ser CNEL EP UN MLG la única empresa distribuidora de energía eléctrica, se apuesta a implemetar una estrategia de operación de subestaciones especialmente aplicada al cantón Marcelino Maridueña, una estrategia que permitirá brindar un sistema eléctrico confiable a nivel de subtransmisión y distribución, ayudando al crecimiento del sector agropecuario e industrial, y garantizando la soberanía alimentaria y el buen vivir con la creación de fuentes de empleo.

\section{Marco Conceptual}

\section{Análisis y evaluación económica -financiera del proyecto}

El análisis y evaluación constan de cuatro divisiones: las inversiones estimadas del proyecto, el financiamiento, los presupuestos (de ingresos y egresos) y los estados financieros pro-forma. Estos cuatro grandes elementos que integran el análisis o estudio financiero y económico de un análisis de factibilidad permitirán reflejar el costo general del proyecto, los ingresos y gastos totales de operación y las fuentes y esquemas de financiamiento que 
requerirá el mismo proyecto. Fuente especificada no válida. Sin duda alguna CNEL EP tendrá que invertir en infraestructura, modernización de equipos de potencia y herramientas de seguridad industrial. El proyecto impactará en el presupuesto anual de operacóon y mantenimiento de subestaciones, el mismo que entrará en consideracion y aprobación de la Agencia de Regulación y Control de Electricidad luego de un análisis económico VAN y TIR que defina si es rentable realizarlo.

\section{Factibilidad Económica}

La factibilidad económica se define mediante un análisis técnicofinanciero para avaluar la factibilidad de realizar y sustentar un proyecto y llevando a cabo también un análisis FODA que deberá realizarse con una recolección de datos, estudios técnicos, ambientales y económicos en la implementación. (Guachún Villalta, 2018). ARCONEL mediante un análisis técnico- financiero cuantificará el objetivo a seguir, y durante el planteamiento como empresa distribuidora tipo estrategia tendrá que visualizar y calcular la proyección de demanda de energía eléctrica a nivel residencial e industrial a niveles de alta tensión. Para tal fin se tuvo que realizar un modelo de operación y mantenimiento para mejorar los niveles de confiabilidad y de igual manera se analizaron las falencias y aciertos de CNLE EP UN MLG.

\section{Análisis Técnicos - Evaluación de campo}

En la actualidad las empresas distribuidoras de energía eléctrica que conforman la Corporación Nacional de Electricidad Empresa Pública - CNEL EP tienen en operación equipos de potencia en obsolescencia (falta de tecnología), presentando problemas en la vinculación con sistemas automatizados, en temas de parametrización de lógica de control, mandos mecánicos y eléctricos. Esta situación conlleva a una actuación lenta e insegura pues su accionamiento debe ser controlado vía local, lo cual lleva de la mano la falta de comunicación entre equipos de control en subestaciones y líneas de subtransmisión y por consiguiente se obtiene el restablecimiento del fluido eléctrico con lentitud, ocasionando malestar en los abonados de tipo residencial, comercial e industrial en media y alta tensión, seguido de daños en equipos por sobre voltaje inducido en el momento del restablecimiento del servicio eléctrico. Este impacto es más considerable en aquellas empresas privadas que no poseen un plan estratégico de operación, aumentando las así las pérdidas técnicas como es el caso particular de las industrias del cantón Marcelino Maridueña.

La subestación Marcelino Maridueña se caracteriza por ser una subestación de paso y reductora, siendo la primera para interconectar las líneas de subtransmisión Milagro 3 y Milagro 4. La primera de ellas suministra a 
nivel de $69 \mathrm{kV}$ las empresas productoras de dicho cantón, la segunda alimenta las subestaciones Tap Bodegas, el Triunfo y Bucay.

\section{Evaluación de proyectos}

La evaluación de proyectos de inversión constituye hoy en día un tema de gran interés e importancia, dado que mediante este proceso se valoran cualitativa y cuantitativamente las ventajas y desventajas de destinar recursos a una iniciativa específica. El análisis de proyecto es un método para presentar el mejor uso de los recursos escasos de la sociedad. Una correcta evaluación hace posible la realización de un proyecto de inversión para que el mismos contribuya al desarrollo a mediano o largo plazo de una empresa en específico.

\section{Características del proyecto}

- Cuentan con un propósito.

- Se resumen en objetivos y metas.

- Plazo de tiempo limitado.

- Cuentan con, al menos, una fase de planificación, ejecución y de entrega.

- Se orientan a la consecución de un resultado.

- Involucran a personas, que actúan en base a distintos roles y responsabilidades.

- Se ven afectados por la incertidumbre.

- Han de sujetarse a un seguimiento y monitorización para garantizar que el resultado es el esperado.

- Cada uno es diferente, incluso de los de similares características.

\section{Subestaciones eléctricas}

Permitir la navegación interactiva del diseño de la subestación, efectuar recorridos virtuales; navegar en todas las áreas de la subestación para ver detalles de los componentes (equipos, materiales, herrajes de conexiones, cables, tubos, buses, conexión a tierra, mandos de cuchillas, barda perimetral, caseta de control, cimentaciones, etc. (Pérez \& otros, 2011). Una subestación eléctrica o centro de transformación es una instalación, o conjunto de dispositivos eléctricos que forman parte de un sistema eléctrico de potencia en donde se modifican los parámetros de tensión y corriente, pasando de niveles de alta, media o baja tensión a niveles adecuados para la distribución de energía eléctrica. Además de esto, estos equipos cumplen la función de unir eléctricamente varios circuitos, siendo el transformador de poder el elemento principal de una subestación.

Entre las principales funciones de un centro de transformación está la de maniobra, protección, supervisión, producción, conversión, 
transformación, regulación, transmisión y distribución de la energía eléctrica, clasificándose en subestaciones de paso, reductoras y elevadoras.

\section{Subestaciones de Paso subestaciones TAP}

Este tipo de subestaciones es la encargada de dar continuidad al servicio mediante un disyuntor que permite abrir y cerrar el circuito eléctrico bajo carga (Voltaje entrada= Voltaje de salida) La línea de subtransmisión Milagro 3 a través de la subestación TAP Bodegas reparte energía a voltajes de alta tensión para los cantones de El triunfo y Bucay.

\section{Subestaciones Reductoras}

Por el contrario, las subestaciones reductoras se encargarán de reducir el voltaje a voltajes de media tensión (Voltaje entrada > Voltaje de salida) Estas subestaciones son constantes y necesarias para reducir el voltaje de niveles de alta a media tensión y así suministrar energía de tipo residencial a través de transformadores de distribución.

\section{Subestación elevadora}

Por último, las subestaciones elevadoras se encargarán de elevar el voltaje (Voltaje entrada < Voltaje de salida) (Nauí, 2015). EMMCA Actualmente CNEL EP UN MLG utilizaba este tipo de subestación ya que permitia elevar el nivel de tensión para transmitir energía a otras subestaciones; sin embargo,actualmente no las posee. 
Figura 1. Diagrama Unifilar - Subestaciones, Líneas de Transmisión y Líneas de

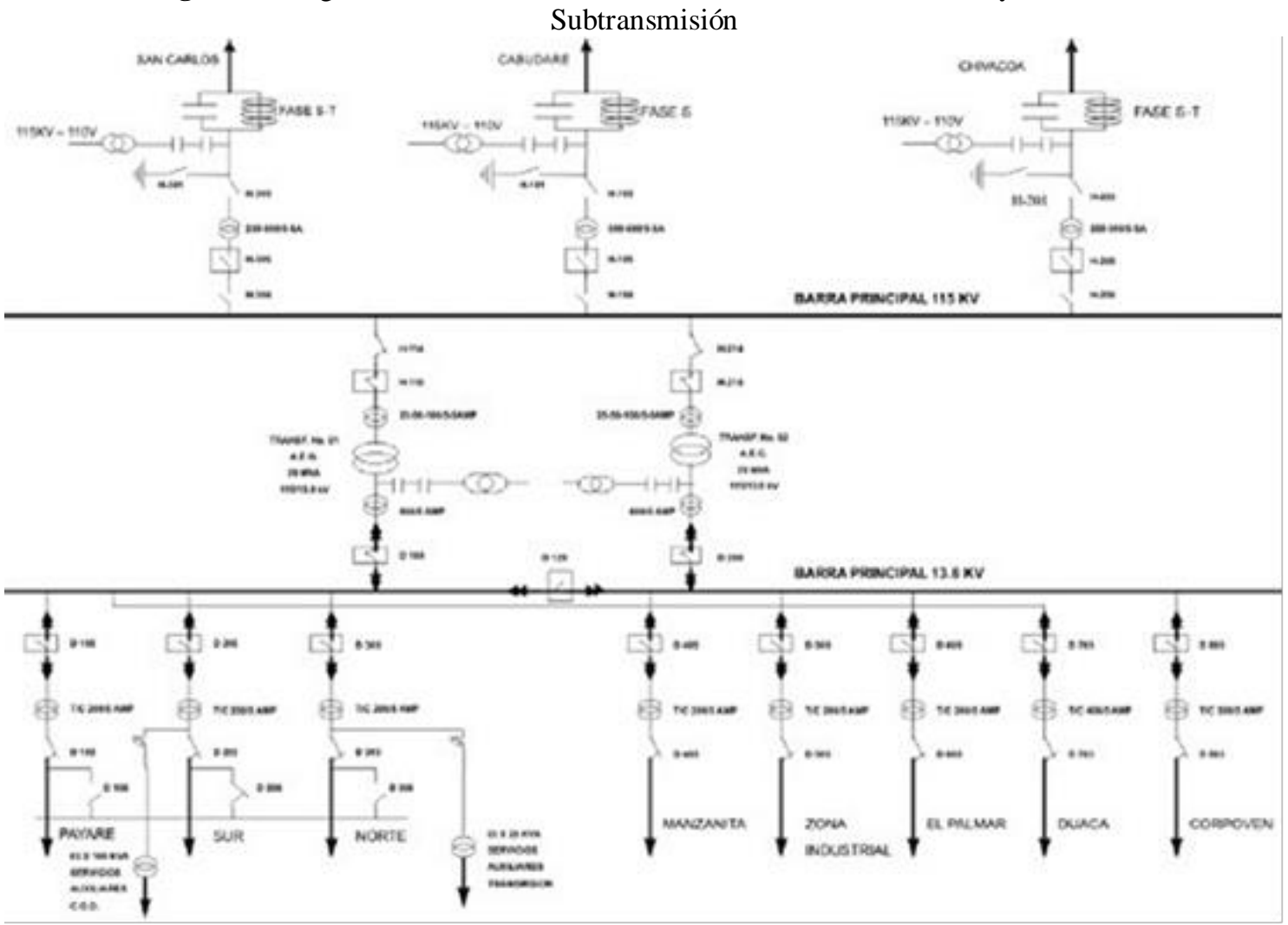

Fuente CNEL EP UN MLG - DEPARTAMENTO DE SUBESTACIONES Y LINEAS DE SUBTRANSMISIÓN.

Elaborado por: Ing. Abel Carrasco Andrade - Topología Eléctrica de CNEL EP MLG.

\section{Equipos de Subestación Eléctrica}

Portafusible: Dispositivo de control de paso de electrones a través de un conductor; medio de protección eléctrico en media tensión para cortocircuitos o sobre cargas de corriente; permite el flujo de electrones siempre que no supere la capacidad permisiva del fusible. Actualmente CNEL EP UN MLG posee 9 fusibles de poder en alimentadoras trifásicas donde la demanda no supera los 2 MW. Estos fusibles deben ser coordinados eléctricamente con el interruptor de barras en media tensión, registrado en el departamento de activos fijos de CNEL EP UN MLG.

Celda: Estructura construida para instalarse de manera auto soportada y destinada a encerrar equipos eléctricos como: transformadores de distribución, transformadores de corriente, transformadores de potencial, equipos de medición y equipos de seccionamiento de media tensión tales como interruptores o seccionadores. Actualmente cuatro subestaciones tienen 
en servicio interruptores encapsulados para protección en media tensión. Cada cuarto de celdas posee 4 salidas trifásicas, 1 interruptor de barras con dimensionamiento a 2000A, registro del departamento de subestaciones y líneas de subtransmisión de CNEL EP UN MLG.

Disyuntores: Interruptor automático térmico - magnético; equipo de protección que permite abrir un circuito eléctrico de media o alta tensión con carga. Como accionamiento mecánico posee un sistema de extinción de arco eléctrico ante un aumento de la intensidad de la corriente o frente a un cortocircuito. Para su accionamiento automático se ayudan de relés de protección. En la Unidad de Negocio Milagro exiten interruptores trifásicos en media y alta tensión. Estos equipos brindan protección de línea y diferencial en las diferentes subestaciones. Dichos equipos poseen una vida útil de 15 años; informacion del Departamento de Planificación.

Gateway: un gateway es un enlace de comunicación entre dispositivos, aplicable en diferentes situaciones; en términos informáticos son nodos en una red que permiten la interconexión de redes con protocolos y arquitecturas. En 3 de 15 subestaciones se tiene implementada la comunicación entre servidores, información otorgada por el Departamento de Informática.

Global positions system (GPS): Sistema de posicionamiento global basado en el espacio, proporcionando un servicio de posicionamiento, navegación y cronometría ininterrumpida a usuarios civiles; este sistema de localización proporcionará su posición y hora con exactitud en coordenadas (latitud, longitud y altitud). Este sistema es de vital importancia dentro del proyecto implementado ya que permite posicionar de manera real las unidades de mantenimiento para realizar maniobras de desconexión.

Patio: Lugar donde se encuentran los equipos de potencia en niveles de media y alta tensión, para maniobra con accionamiento mecánico eléctrico. Dentro de las 15 subestaciones de CNEL EP UN MLG, se tienen dos patios de maniobras que constan de equipos de potencia.Es también un área necesaria para la maniobra de vehículos y el mantenimiento correctivo de equipos.

Reconectador: Dispositivo de interrupción que funciona bajo carga; cuenta con secuencia de cierre mecánico inmerso en aislamiento al igual que al vacío, en aceite o en SF6. Un reconectador con parámetros IED permite monitorear los parámetros de red. Este equipo eléctrico estático trabaja con energía alterna y contínua, Para el circuito de fuerza y de control respectivamente, sirve para despejar una falla con corrientes altas en períodos de tiempo muy pequeños.

Fuentes de energía renovable: la energía renovable es el tipo de energía obtenida por fuentes naturales, clasificadas en energías renovables tipo convencionales y no convencionales. Entre las primeras se encuentran las 
grandes hidroeléctricas y entre las segundas se denotan las térmicas, geotérmicas, mareomotriz, biomasa pequeñas hidroeléctricas. (Spiegeler \& Cifuentes). Particularmente las empresas privadas tales como los ingenios azucareros reutilizan sus desechos con el fin de crear fuentes de energía limpia, energía que es vendida al Sistema Nacional de Electricidad a tasas establecidas por el ente rector de comercialización.

SCADA (Supervisión, Control y Adquisición de Datos): es una aplicación software diseñada para supervisar y controlar a distancia sistemas de operación basados en procesos; utiliza como medio de comunicación controladores lógicos programables (PLC). El SCADA permite monitorear un proceso de forma sistematizada, es decir, vía remoto en tiempo real, y es utilizado como herramienta para realizar estudios de posibles fallas, generar alarmas, diagnósticos de equipos y toma de decisiones.

\section{Automatizacion subestaciones electricas}

Actualmente el Sistema de Automatización de las subestaciones eléctricas (SAS), es un proceso enfocado principalmente a la correcta operación, funcionalidad e interoperabilidad de los equipos que conforman la subestación eléctrica para que de esta manera las acciones puedan ser realizadas en el menor tiempo posible y con la precisión necesaria.

Estos equipos son integrados en sistemas SCADA a través de diversos protocolos. Los sistemas de control de las subestaciones eléctricas, desde el punto de vista del control y automatización, están por lo general divididos en 3 niveles de automatización:

Nivel 1: corresponde a los equipos de posición, los cuales están conectados directamente al nivel 0 y realizan funciones de protección, control y adquisición de datos, mediante lógica programable - equipos con función IED. Tres de 15 subestaciones poseen este nivel de integración de acuerdo al área de SCADA de CNEL EP UN MLG.

Nivel 2 :realiza funciones de control, supervisión y adquisición de datos de todos los equipos de posición y de integración de equipos de potencia al sistema SCADA. Su enlace se da en el centro de control de operaciones. Este monitoreo se realiza a través de una plataforma georeferenciada en tiempo real.

Nivel 3 : se realiza la comunicación de equipos y también se realiza la operatividad - monitoreo del proceso. (Avella \& Andrés, 2016). El tipo de comunicación es por DNP3; no hay integración por fibra óptica.

\section{Metodología}

Los estudios analíticos, sintéticos o estudios de casos se subdividen en dos grandes grupos; el primero partiendo del estudio sobre hechos o descomposición del objeto de estudio en cada una de sus partes para 
estudiarlas en forma individual (análisis), y luego se integran esas partes para estudiarlas de manera holística e integral (síntesis), mientras que el segundo consiste en estudiar a profundidad o en detalle una unidad de análisis específica, tomada de un universo poblacional. El caso o unidad de análisis puede ser una persona, una institución o empresa, un grupo, etcétera. Las principales fuentes para la obtención de la información son las personas directamente relacionadas con el caso o la unidad de análisis y documentos de toda índole válidos que contengan información sobre el caso. Fuente especificada no válida.

La subestación Marcelino Maridueña analizada como Centro de Reducción de Voltaje posee una cargabilidad de $41 \mathrm{MW}$ en condiciones normales con transformadores de poder trabajando al $85 \%$ bajo condiciones de circulación sin ventilación forzada. Esta cargabilidad representa un valor mensual de \$1,328.400.00 dólares a un valor de 9 ctvs. En calidad de subestación de paso posee una cargabilidad de 10.2 MW, alimentando a empresas como Papelera Nacional, Ingenio San Carlos y Soderal, clientes que son considerados como industriales. Esta cargabilidad representa un valor a facturar de $\$ 330.480 .00$.

Además, la misma subestación Marcelino Maridueña es analizada como Centro de Transferencia de Voltaje de paso, y brinda energía a la compañía azucarera Ingenio San Carlos. Se analizará la posibilidad de disminuir el tiempo de operación al efectuar el cambio de alimentación Milagro 3 y Milagro 4, implementando un sistema de eliminación de armónicos - transitorios eléctricos - filtro paso bajo, equipo que protegerá de las variaciones de voltaje y frecuencia, a los equipos electrónicos de Papera Nacional y Soderal.

San Carlos siendo una empresa industrial del tipo cañicultor que posee una cargabilidad de transferencia de $13 \mathrm{MW}$, energía producida por medio debiomasa mediante quema de desecho orgánico. Este tipo de suminstro de energía es planificada y estructurada mediante energía renovable rentable, aplicando un sistema de estructuración no convencional. 


\section{Analisis de datos y curvas generadas}

Tabla 1. Análisis de Compra de Energía a Generadoras tipo Hidroelecrica.

Histórico de Distribución Porcentual de Compra de Energía en el Mercado Ocasional y

Contratos $2011-2017$.

\begin{tabular}{cccccc} 
PERIODO & $\begin{array}{c}\text { ENERGIA CONTRATOS } \\
\text { REGULAD OS (kWh) }\end{array}$ & $\begin{array}{c}\text { ENERGIACONTRATOS } \\
\text { PRIVADOS (kWh) }\end{array}$ & $\begin{array}{c}\text { ENERGIA MERCADO } \\
\text { OCASIONAL (kWh) }\end{array}$ & $\begin{array}{c}\text { ENERGIA CONTRATOS } \\
\text { REGULAD O5 }\end{array}$ & $\begin{array}{c}\text { ENERGIAMERCADO } \\
\text { OCASIONAL }\end{array}$ \\
\hline 2011 & 5901723655 & 8472867,49 & 746225480,7 & $88,66 \%$ & $11,21 \%$ \\
2012 & 6213567938 & 8296985,67 & 329056119,1 & $94,85 \%$ & $5,02 \%$ \\
2013 & 6333304546 & 7964894,64 & 569353561,9 & $91,65 \%$ & $8,24 \%$ \\
2014 & 10731330014 & 7312828,18 & 1137903068 & $90,36 \%$ & $9,58 \%$ \\
2015 & 11804463015 & 8409752,407 & 1297209498 & $90,04 \%$ & $9,89 \%$ \\
2016 & 14487295519 & 9141035,225 & 1456515928 & $90,81 \%$ & $9,13 \%$ \\
2017 & 15560428520 & 9965556,602 & 1581685265 & $90,72 \%$ & $9,22 \%$
\end{tabular}

SISDAT-CONELEC

Elaborado por: Ing. Abel Carrasco Andrade - Datos Estadísticos ARCONEL

Figura 2. Histórico de Porcentaje de Compra de Energía 2011 - 2018.

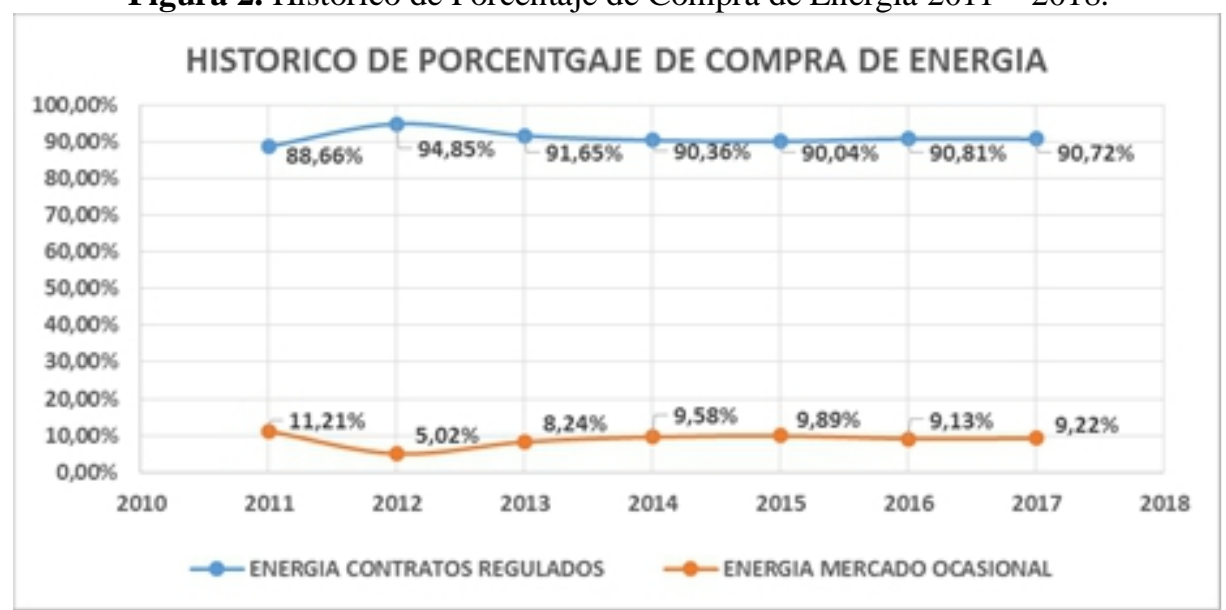

Fuente: SISDAT-CONELEC

Elaborado por: Ing. Abel Carrasco Andrade - Datos Estadísticos ARCONEL

\section{Mercado de Contratos - Energía Eléctrica}

A partir de la estabilidad de suministro de energía eléctrica que se ha venido dando en el periodo 2012 a 2018 con respecto a la compra de energía eléctrica, estabilización que coincide con la creación de la línea Milagro 3 y la independencia de carga industrial, se puede inducir notablemente que el usuario no confía en el sistema eléctrico de potencia que proporciona CNEL EP UN MLG.

Estudio técnico a un transformador de tensión con núcleo de potencia, usado para la alimentación de los servicios auxiliares de una subestación tipo maniobra, buscando mitigar la posibilidad de perder la continuidad del suministro de energía eléctrica y asimismo evita la dependencia del agente 
distribuidor externo (Viteri Toquica Diego1, 2016). La subestación Marcelino Maridueña, posee equipos de potencia de patio a $69 \mathrm{kV}$; consta de un disyuntor a $69 \mathrm{kV}$, tipo GIS, cuyo medio de aislamiento es en vacío. Este interruptor le otorga la cualidad de ser subestación de paso suministrando energía a 69 kv a otras subestaciones y al mismo tiempo sirve de protección para el transformador de poder. Esta última cualidad permite alimentar al transformador de poder para suministrar voltaje a nivel de distribución, con sus debidas salidas de alimentadores mediante celdas tipo encapsuladas Celdas Metalclad. El costo de la presente subestación corresponde a $\$ 3,089.318 .01$.

Tabla 2. Indicadores de desconexión de energía de CNEL EP UN MLG.

\begin{tabular}{|l|l|l|}
\hline $\begin{array}{l}\text { AÑO } \\
\text { INDICADOR }\end{array}$ & FMIK & TTIK \\
\hline 2010 & 43,39 & 47,91 \\
\hline 2011 & 37,27 & 43,36 \\
\hline 2012 & 34,69 & 41,2 \\
\hline 2013 & 30,32 & 36,33 \\
\hline 2014 & 24,29 & 26,04 \\
\hline 2015 & 10,98 & 9,59 \\
\hline 2016 & 7,34 & 9,16 \\
\hline 2017 & 6,85 & 6,99 \\
\hline 2018 & 6,63 & 6,69 \\
\hline
\end{tabular}

Fuente: SISDAT-CONELEC

Elaborado por: Ing. Abel Carrasco Andrade - Datos Estadísticos CNEL EP UN MLG

Figura 3. FMIK y TTIK

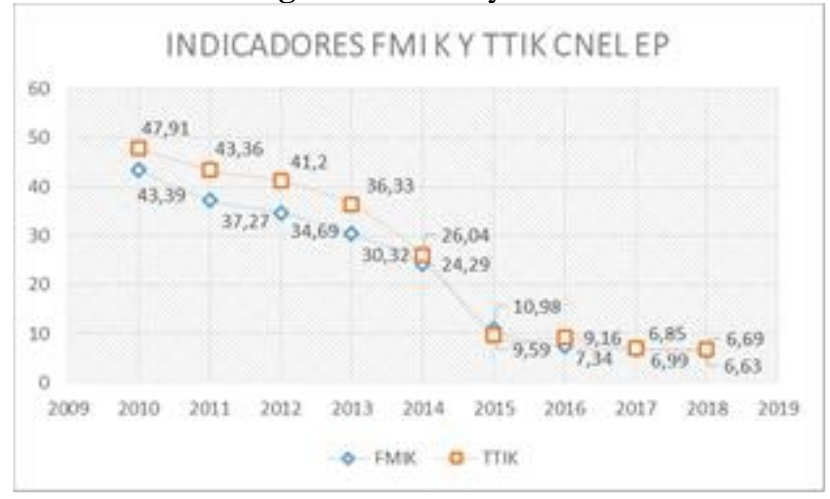

Fuente: CNEL EP INDICADORES FMIK Y TTIK

Elaborado por: Ing. Abel Carrasco Andrade - Datos Estadísticos CNEL EP UN MLG

De acuerdo al histórico de desconexiones de líneas de Subtransmisión suministrado por el departamento de Planificación de CNEL EP UN MLG se puede apreciar que el índice de desconexión en el período comprendido entre 
2009 y 2015 tiene un índice notable con una pendiente de 6.15, período en el cual se crearon líneas de subtransmisión como Milagro 3, exclusiva para suministrar energía a las industrias como SODERAL, INGENIO SAN CARLOS Y PAPELERA NACIONAL. Del período 2015 al 2018 las desconexiones se han estabilizado debido al transiente eléctrico que se produce en el momento de transferencia de energía eléctrica entre Milagro 3 y Milagro 4.

Tabla 3. Recaudaciones deCNEL EP UN MLG

\begin{tabular}{|l|l|}
\multicolumn{1}{c}{ AÑO / } & \multicolumn{1}{c}{$\%$} \\
INDICADOR & RECAUDACIÓN \\
\hline 2010 & $88,95 \%$ \\
\hline 2011 & $94,85 \%$ \\
\hline 2012 & $99,94 \%$ \\
\hline 2013 & $99,02 \%$ \\
\hline 2014 & $98,77 \%$ \\
\hline 2015 & $98,06 \%$ \\
\hline 2016 & $97,68 \%$ \\
\hline 2017 & $99,76 \%$ \\
\hline 2018 & $99,65 \%$ \\
\hline
\end{tabular}

Fuente: CNEL EP INDICADORES FMIK Y TTIK

Elaborado por: Ing. Abel Carrasco Andrade - Datos Estadísticos CNEL EP UN MLG

Figura 4. Clientes

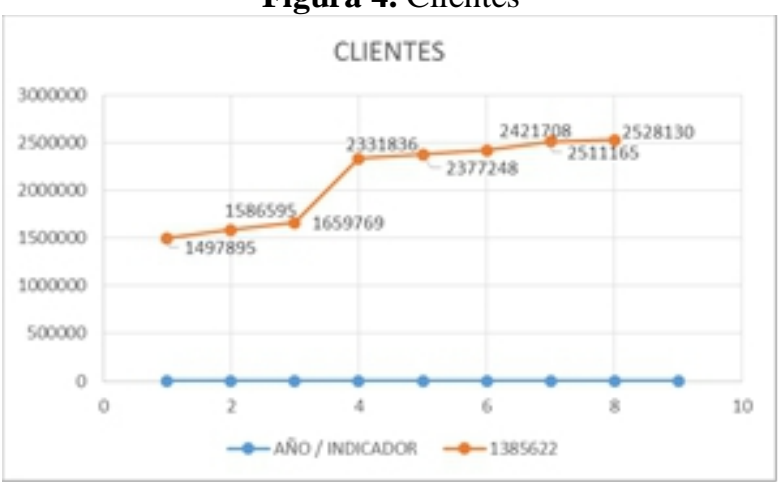

Fuente: CNEL EP UN MLG CLIENTES

Elaborado por: Ing. Abel Carrasco Andrade - Datos Estadísticos CNEL EP.

El incremento de clientes y el porcentaje de recaudación entre los años 2011 y 2018, coincide con el período donde la subestación Marcelino Maridueña divide el suministro de carga, dando a empresas industriales el abastecimiento exclusivo por la línea de subtransmisión Milagro 3. Considerando el actual proyecto es posible indicar que al momento de poder 
transferir carga entre Milagro 3 y Milagro 4, se podrá incrementar el número de clientes de acuerdo a la confiabilidad del sistema eléctrico de potencia.

Los estudios descriptivos buscan especificar las propiedades, las características y los perfiles de personas, grupos, comunidades, procesos, objetos o cualquier otro fenómeno que se someta a un análisis. Es decir, únicamente pretenden medir o recoger información de manera independiente o conjunta sobre los conceptos o las variables a las que se refieren; esto es, su objetivo no es indicar cómo se relacionan éstas. Fuente especificada no válida. En la subestación Marcelino Maridueña se buscó especificar características, perfiles, procedimentos, operación y funcionabilidad de equipos, recopilando información mediante medición de tasas de fallas y años de servicio e identificando las variables relacionadas con la descripción del problema, las cuales son la poca confiabilidad del sistema eléctrico de potencia y la operación de equipos para mejorar los tiempos de respuesta a desconexiones del suministro de energía.

Consiste en un análisis de la información escrita sobre un determinado tema, con el propósito de establecer relaciones, diferencias, etapas, posturas o estado actual del conocimiento respecto al tema objeto de estudio. Las principales fuentes de información en este tipo de investigación son: documentos escritos (libros, periódicos, revistas, actas notariales, tratados, conferencias escritas, etcétera), documentos fílmicos (películas, diapositivas, etcétera) y documentos grabados (discos, cintas, casetes, disquetes, etcétera). Fuente especificada no válida. Como método de análisis descrito se tuvo que identificar y analizar información escrita sobre parámetros, tipos de subestaciones, implementación de monitoreo de señales eléctricas, identificando así etapas del proceso y el objeto del análisis.

Figura 5. Proceso de Generación de Energía Eléctrica y Transferencia.

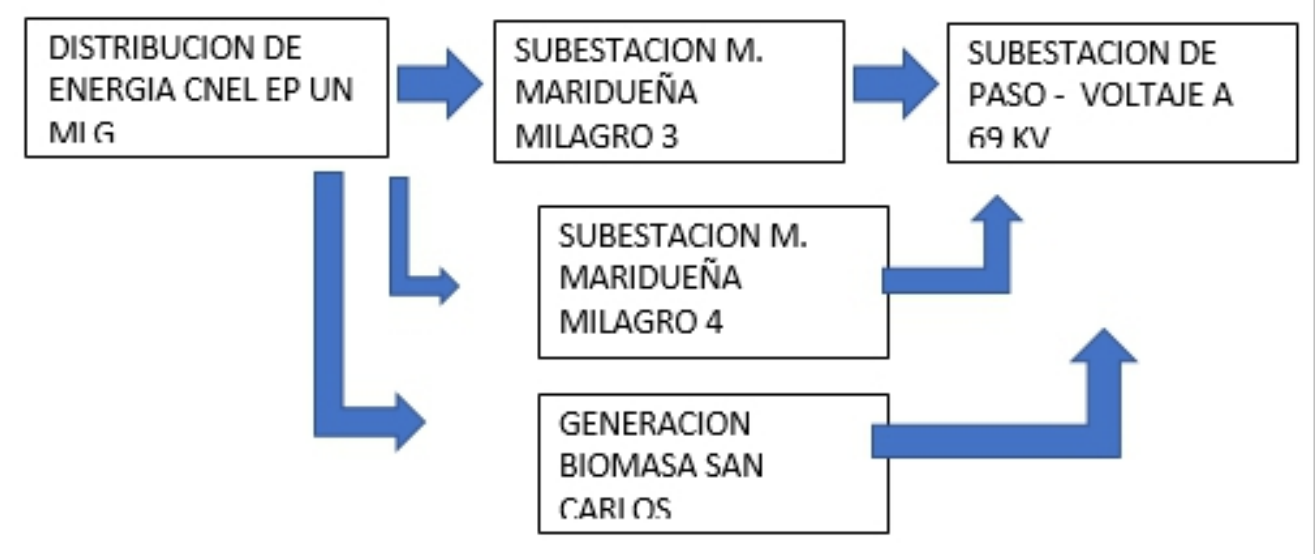

Fuente: PROCESO DE SUMINISTRO DE ENERGIA

Elaborado por: Ing. Abel Carrasco Andrade - Datos Estadísticos CNEL EP 


\section{FODA}

Desarrollo tanto socialmente como económicamente cumpliendo con todas las normas de la construcción post-terremoto, cumpliendo normas ambientales (Valarezo Vera \& Genith Maria, 2018). CNEL EP UN MLG implementará el proyecto con diseños, implantaciones de equipos, obra civil y adquisición de equipos para un grado de sismicidad mayor o igual a 0.5, factor importante para la compra de un transformador de poder el cual es considerado como el corazón de una subestación eléctrica. Por el motivo antes expuesto se necesitó realizar un análisis FODA de la topología eléctrica de la subestación Marcelino Maridueña.

FORTALEZAS

UNICO SUMINSTRO DE ENERGLA ELECTRICA A NIVEL DE SUETRANSMISION.

transferencia de energia entre liNeA de SUBTRANSMISION MILAGRO 3 Y MILAGRO 4.

LECTURA DE PARAMETROS ELECTRICOS EN TIEMPO REAL.

espacio fisico NEcesario para REMODELACION DE SUBESTACION SIN AFECTAR LACONINUIDAD DEL SERVICIO

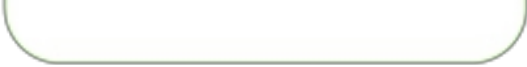

\section{DEBILIDADES}

OISYUNTOR SUBESTACION DE PASO OBSOLETO. OPERATIVO.

celoAs de MEOIA TENSION OBSOLETAS - operativas INTERCONEXION DE LA LINEA DE SUBTRANSMISION MILAGRO 3 Y MILAGRO 4 CON FLUCTUACION DE VOLTAE

DESCONEXION DEL SISTEMA ELECTRICO POR TRANSFERENCIA DE ENERGIA ENTRE LINEAS DE SUBTRANSMISION MILAGRO 3 Y MILAGRO 4 no garantiza la continuidad de Servicio en la TRANSFERENCIA DE ENERGIA

\section{(n)

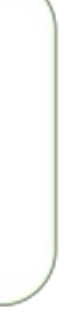
OPORTUNIDADES
REEMPLAZO de EQUIPOS de POTENCIA A
NIVEL DE SUBTRANSMISION
REEMPLAZO DE EQUIPOS DE POTENCIA EN MEOLA TENSION
IMPEMENTACION DE UNA PLATAFORMA
VIRTUAL DE MONITOREO DE PARAMETROS ELECTRICOS EN TIEMPO REAL
REMODELACION DE SUBESTACION PATIO DE MANIOBRAS E IMPLEMENTACION DE EQUIPO PARA ELIMINACION DE TRANSITORIOS ELECTRICOS

\section{OPORTUNIDADES}
perdóda de sumintro de energia A EMPRESAS INDUSTRIALES A NIVEL DE SUBTRANSMISION
TRANSELECTRIC POORIA SUMINISTRAR ENERGIA A NIEVES DE TRANSMISION
IMPEMENTACION DE UNA PLATAFORMA VIRTUAL DE MONITOREO DE PARAMETROS ELECTRICOS EN TIEMPO REAL.
- SUMINISTRO de ENERGLA A LA UNEA de SUBTRANSMISION MILAGRO 3 MEDIANTE ENERGLA RENONABLE

\section{Gráfico 5. Fuente: ANALISIS FODA CNEL EP UN MLG SUBESTACION MARECELINO MARIDUEÑA OPERACIÓN VIGENTE.}

Elaborado por: Ing. Abel Carrasco Andrade - Datos Estadísticos CNEL EP

Gracias al análisis FODA, podemos evidenciar que la empresa al ser gubernamental del tipo estratégica tiene ventajas en la comercialización y suministro de energía eléctrica a niveles de $69 \mathrm{kV}$, sabiendo que existe una debilidad impresionante porque no presenta una confiabilidad del sistema eléctrico de potencia para sus abonados de tipo industrial. Es vital hacer énfasis en las oportunidades notables que ofrece CNLE EP a través de la subestación Marcelino Maridueña. Las redes de alimentación en niveles de subtransmision son propias, con opción a transferir carga entre Milagro 3 y Milagro 4. Es por ello que se analiza la posibilidad de implementar una estrategia de operación desde la subestación Marcelino Maridueña.

La investigación generó una estrategia que busca contribuir a fortalecer la producción y comercialización de amaranto. La estrategia se 
generó con la metodología de marco lógico utilizando información recopilada (Romero Romano \& Carlos Osvaldo, 2018). El árbol de problemas es una metodología utilizada para detectar los problemas y efectos del sistema de operación vigente - caso Subestación Marcelino Maridueña; así con este análisis podemos identificar la causa real del problema.

Implementación de algoritmos para solucionar problemas, definiéndolo, analizando modelo para resolución (Acero, 2018). Implementando el modelo de Tompson debemos establecer tareas con el objetivo de desarrollar un plan estratégico viable según el modelo del negocio, ya que CNEL EP UN MLG, siendo una empresa estratégica, define su misión y visión de la empresa. De igual forma, al enfocarse en el punto de análisis se evidencia que el objetivo es suministrar energía eléctrica a niveles de subtransmisión con confiablidad y continuidad de servicio sin desconexiones de índole técnico.
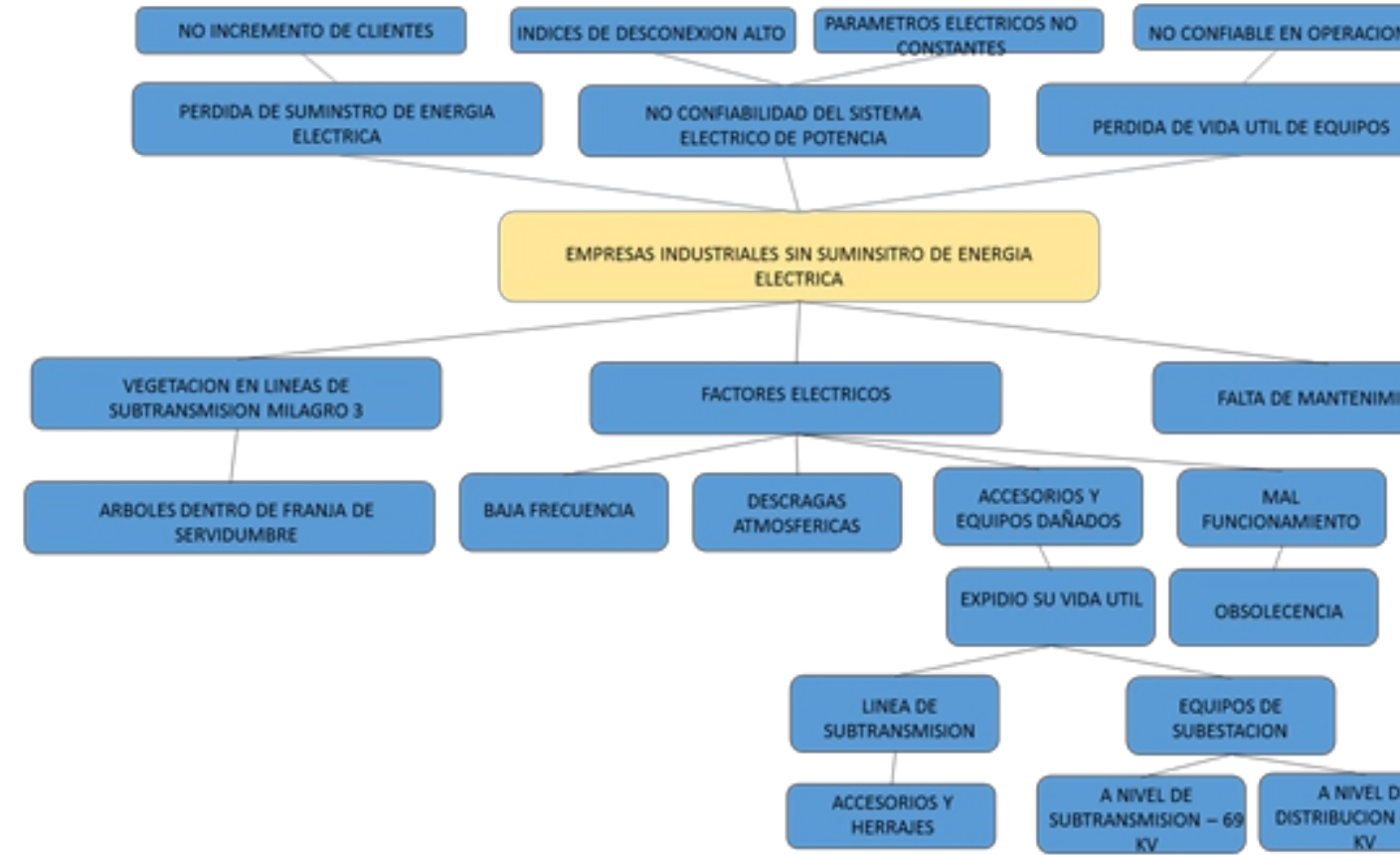

\section{Gráfico 5. ANÁLISIS CAUSA Y EFECTO CNEL EP UN MLG SUBESTACIÓN MARECELINO MARIDUEÑA OPERACIÓN VIGENTE.}

Elaborado por: Ing. Abel Carrasco Andrade - Datos Estadísticos CNEL EP

Para disminuir los efectos y las causas de la incidencia de la desconexión por factores eléctricos y falta de mantenimiento, lo primero que se busca hacer es implementar un equipo para eliminar desconexiones por transitorios eléctricos producidos por descargas atmosféricas e interconexión entre las líneas de subtransmisión Milagro 3 y Milagro 4, mientras que la 
segunda acción a tomar esreemplazar equipos eléctricos que han terminado su vida útil como es el caso del reemplazo de los equipos eléctricos de la subestación Marcelino Maridueña.

La implementación de un modelo de gestión de proceso para un sistema electrico automatizado y controlado remotamente se realiza mediante la evaluación técnica - económica de un reporte cualitativo y cuantitativo de ventajas y desventajas en la aplicación de políticas de mantenimiento de tipo predictivo, preventivo y correctivo, apegado a un análisis de fortalezas y debilidades.

El éxito de los proyectos ha sido medido tradicionalmente en términos del cumplimiento del presupuesto y el cronograma. Sin embargo, la ejecución de los proyectos integra personas internas o externas a la organización para alcanzar unos objetivos específicos. (ARIZA D.A, 2017). El proyecto a implementar en la subestación Marcelino Maridueña satisface los parámetros de confiabilidad de un sistema eléctrico de potencia ejecutable en el tiempo y que puede ser sustentable económicamente. Al ser una empresa de carácter público debe tener un valor agregado tomando en cuenta la inversión inicial y cuán sustentable va a ser a través de los años, presentando un organigrama que ayude al cumplimiento de los objetivos específicos.

\section{Estrategia de operación - Implementación Subestación Marcelino Maridueña}

La investigación se desarrolló en el ámbito del sector construcción, y buscará conocer los resultados que se obtienen al aplicar la metodología de la ingeniería de valor en la administración del proyecto de Saneamiento de Sistemas Operativos - EPC (Pineda Bernabel \& Ronald David, 2017). Por ello este análisis se enfoca en diseñar una estrategia de operación ideal que implica el suministro, confiabilidad y calidad de servicio con el objetivo de cumplir la misión y visión del proyecto alineado a la de la Corporación Nacional de Electricidad Unidad de Negocio Milagro. Se detalla así a continuación la estrategia de operación de la Subestación Marcelino Maridueña. 
ESTRATEGIA DE OPERACIÓN SUBESTACION MARCELINO MARIDUEÑA

- Calidad de servicio Interconexión del sistema de Subtransmision.

- Eliminación de Fluctuaciones de Voltaje.

- Confiablidad del sistema de Subtransmision ante desconexiones por fluctuaciones de voltaje

$\Rightarrow$

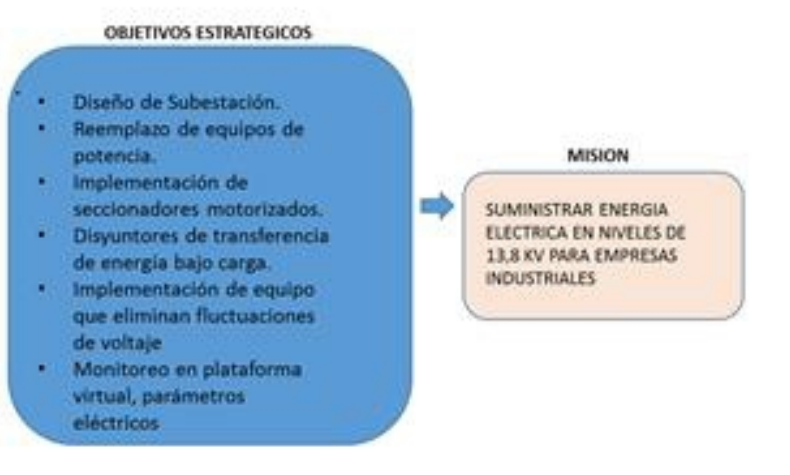

Gráfico 6. ESTRATEGIA DE OPERACIÓN A IMPLEMENTAR

Elaborado por: Ing. Abel Carrasco Andrade - Datos Estadísticos CNEL EP

Una vez detectada la estrategia de operación, identificamos la misión del proyecto el cual es dirigido a abarcar los objetivos creados tanto como objetivo general como específicos. A continuación se detalla el diagrama de procesos a implementar:

\section{Secuencia de Operación Propuesta}

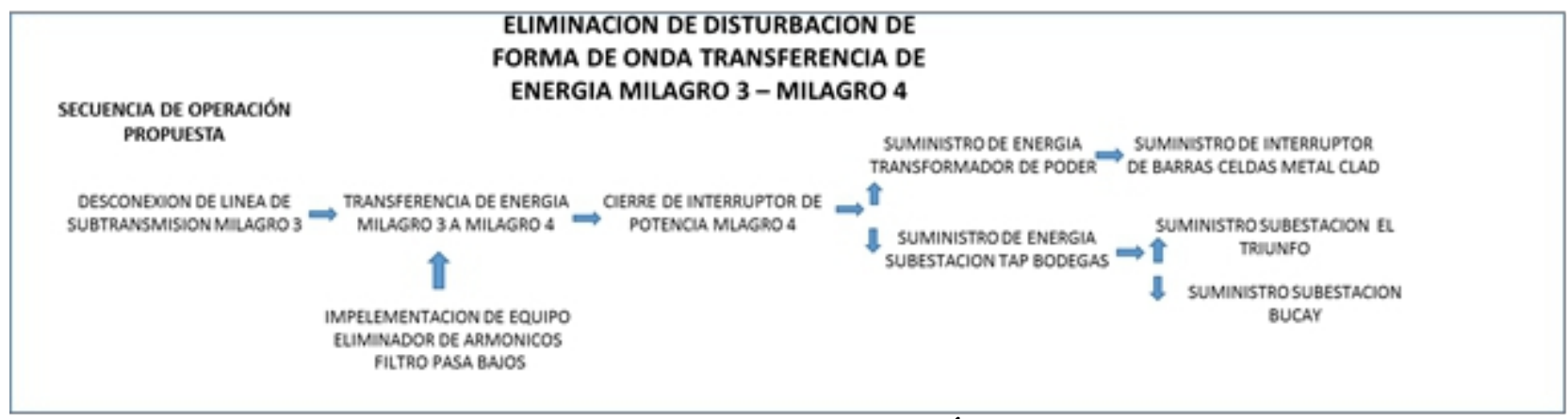

\section{Gráfico 7. SECUENCIA DE OPERACIÓN PROPUESTA}

Elaborado por: Ing. Abel Carrasco Andrade - Datos Estadísticos CNEL EP

Para la debida ejecución delpresente esquema se necesita la implementación de un sistema interconectado que incluirá un patio de maniobra a $69 \mathrm{kV}$, con seccionadores motorizados de entrada y salida de disyuntores de transferencia con un BIL de $72 \mathrm{kV}$, implementando también un sistema de eliminación de pasa bajos a nivel de la subtransmisión para eliminar la distribución de forma de ondas que implica una desconexión de suministro por armónicos. 


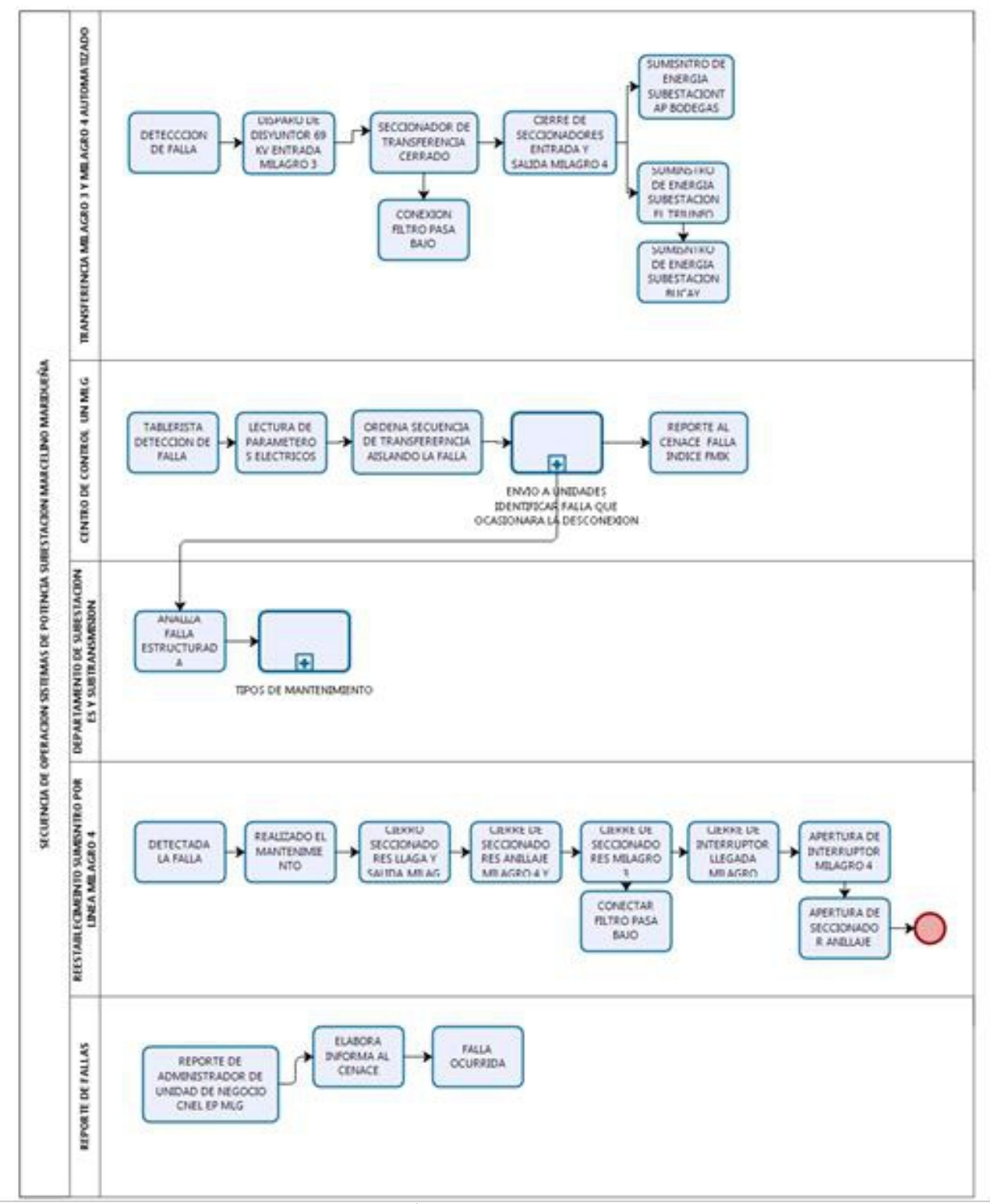

\section{Gráfico 8. ESQUEMA DE OPÉRACION PROPUESTO CNEL EP UN MLG SUBESTACIÓN MARECELINO MARIDUEÑA OPERACIÓN VIGENTE}

Elaborado por: Ing. Abel Carrasco Andrade - Datos Estadísticos CNEL EP 


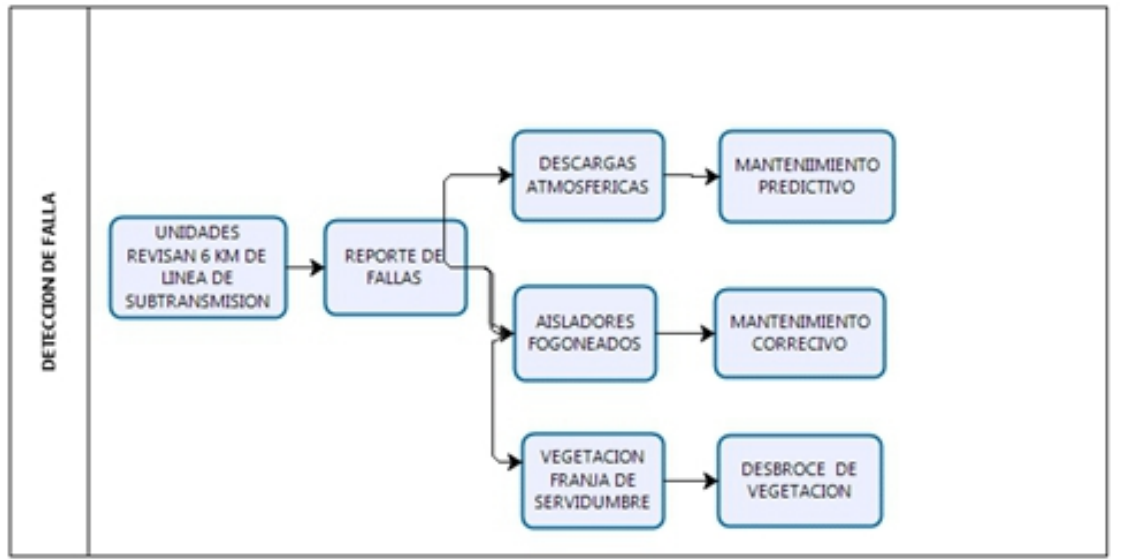

Gráfico 9. DETECCIÓN DE FALLA- SECUENCIA DE OPERACIÓN PROPUESTA CNEL EP UN MLG SUBESTACION MARECELINO MARIDUEÑA OPERACIÓN VIGENTE

Elaborado por: Ing. Abel Carrasco Andrade - Datos Estadísticos CNEL EP

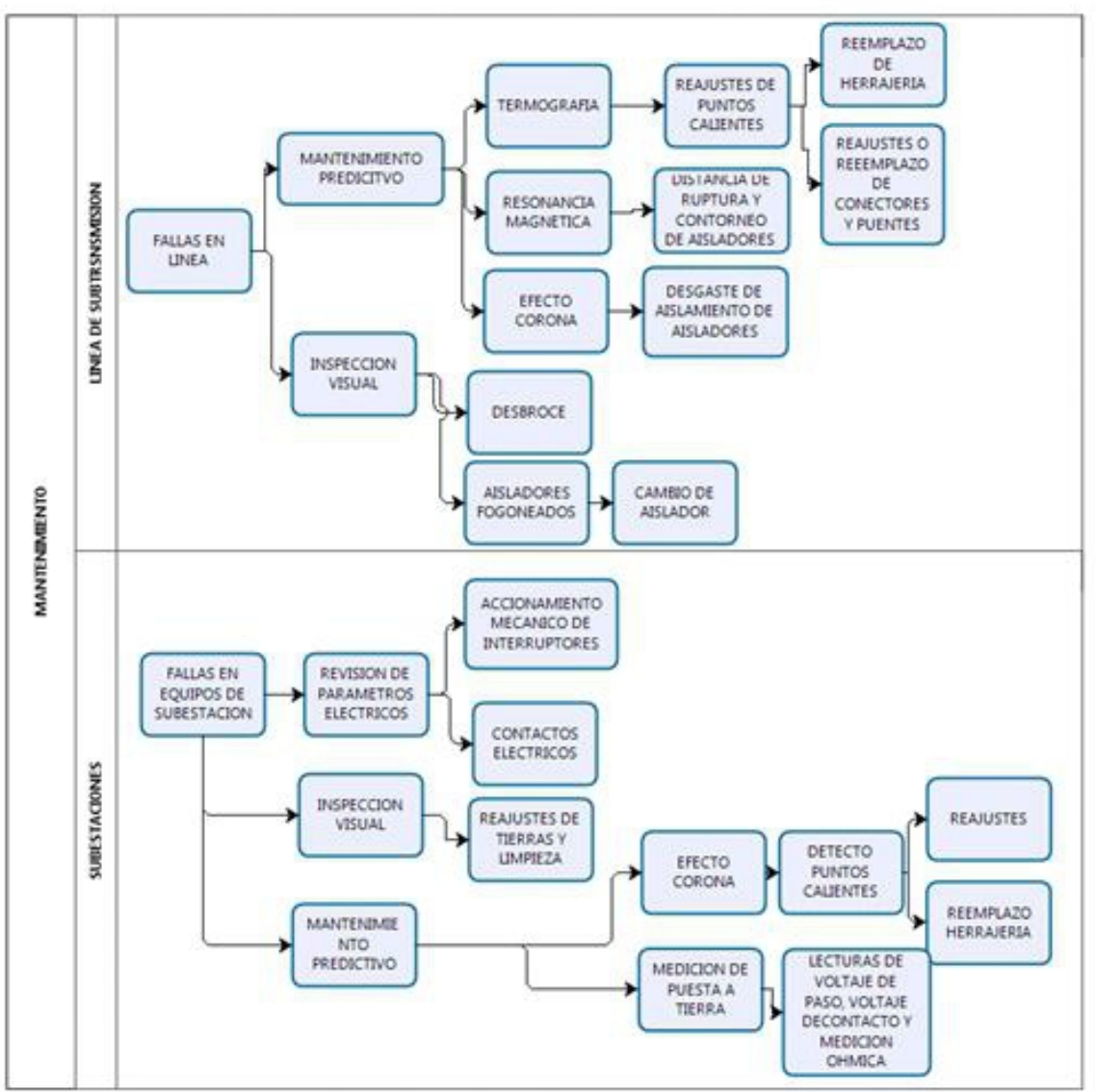

Gráfico10. MANTENIMIENTO PROPUESTO CNEL EP UN MLG SUBESTACION MARECELINO MARIDUEÑA OPERACIÓN VIGENTE

Elaborado por: Ing. Abel Carrasco Andrade - Datos Estadísticos CNEL EP 
Cálculos de Rentabilidad y Valor Actual Neto

INDICADORES FINANCIEROS Y ECONOMICOS

\begin{tabular}{|c|c|c|c|c|c|c|}
\hline NNo. & Nom bre del Proyecto & $\begin{array}{l}\text { CLIENTES } \\
\text { POTENCIALES }\end{array}$ & $\begin{array}{c}\text { Costo } \\
\text { Promedio } \\
\text { venta kwh }\end{array}$ & $\begin{array}{l}\text { Consumo total } \\
\text { Promedio kwh }\end{array}$ & $\begin{array}{l}\text { \$Costo } \\
\text { Proyecto }\end{array}$ & $\begin{array}{c}\text { COBUMD } \\
\text { QIENES } \\
\text { POIEVAALs }\end{array}$ \\
\hline 1 & $\begin{array}{l}\text { REPOTENCACCON YRETALRACON } \\
\text { DE INFRAESTRUCULA SLBESTACON } \\
\text { MARCBINOMARIDUENA 18/24 MVA. }\end{array}$ & 3 & 0,085 & $4.080,00$ & $1.304 .688,00$ & $16.000,00$ \\
\hline 2 & $\begin{array}{c}\text { APLIOANDOEERGA RENDVABE- } \\
\text { BSOMASA }\end{array}$ & 3 & 0,095 & $4.560,00$ & - & $16.000,00$ \\
\hline & $\begin{array}{l}\text { Costo de Compra al SISTEMA } \\
\text { NACIONAL INIERCONECTADO }\end{array}$ & 0,0461 & & & & \\
\hline
\end{tabular}

\begin{tabular}{|c|c|}
\hline \multicolumn{2}{|l|}{ FACTORES ENERGETICOS } \\
\hline Tasa de Descuento & $0 \%$ \\
\hline Inversión Total (USD) & $\$ 1.304 .688,00$ \\
\hline Consumo KW-h/mes Usuario Nuevo & $16.000,00$ \\
\hline Consumo KW-h/mes Usuario Antiguo & $10.000,00$ \\
\hline Costo por demanda sustituta USD/mes & $4.560,00$ \\
\hline Energia no suministrada a usuario $\mathrm{KWh} /$ usuario - pedidas te & 800 \\
\hline
\end{tabular}

\begin{tabular}{|l|r|}
\hline \multicolumn{2}{|c|}{ Indicadores Económicos (Sociales) } \\
\hline VAN & $11.433 .556,68$ \\
\hline TIR & $17 \%$ \\
\hline RBC & 22,93 \\
\hline El Programa & Es viable \\
\hline
\end{tabular}

\begin{tabular}{|l|c|}
\hline \multicolumn{2}{|c|}{ VIDA ÚTIL DE LOS PROYECTOS POR ETAPAS FUNCIONALES } \\
\hline Etapa Funcional & Vida Útil (años) \\
\hline Equipos de potencia & 35 \\
\hline Transformadores de Distribución & 30 \\
\hline Redes Secundarias & 35 \\
\hline Alumbrado Publico & 25 \\
\hline Acometidas y Medidores & 20 \\
\hline Instalaciones Civiles Generales & 10 \\
\hline
\end{tabular}

Fuente Dirección de Tarifas ARCONEL

\begin{tabular}{|l|c|}
\hline \multicolumn{2}{|c|}{ COSTOS DE OPERACIÓN Y MANTENIMIENTO } \\
\hline Etapa Funcional & Indices (\%) \\
\hline Sistema deTransmisión & 3 \\
\hline Líneas de Subtransmisión & 3 \\
\hline Subestaciones de Distribución & 4 \\
\hline Alimentadores Primarios & 6 \\
\hline Transformadores de Distribución & 9 \\
\hline Redes Secundarias & 9 \\
\hline Alumbrado Público & 9 \\
\hline \multicolumn{2}{|l|}{ Fuente Dirección de Tarifas CONELEC } \\
\hline
\end{tabular}




\begin{tabular}{|c|c|c|c|c|c|c|c|}
\hline \multicolumn{8}{|c|}{ EVALUACION ECONOMICA } \\
\hline \multirow[b]{2}{*}{ Años } & \multirow[b]{2}{*}{ Inversión } & \multicolumn{4}{|c|}{ Ingresos } & \multirow{2}{*}{$\begin{array}{l}\text { Egresos } \\
\text { Compra } \\
\text { energia }\end{array}$} & \multirow[b]{2}{*}{ Flujo Neto } \\
\hline & & $\begin{array}{c}\text { Venta de } \\
\text { Energia }\end{array}$ & $\begin{array}{c}\text { Ahorro al no } \\
\text { realizar } \\
\text { remodelacion } \\
\text { de } \\
\text { subestacion } \\
\text { M. Maridueña }\end{array}$ & $\begin{array}{c}\text { Incremento de } \\
\text { energía vendida } \\
\text { por disminución } \\
\text { en tiempos de } \\
\text { interrupción }\end{array}$ & $\begin{array}{l}\text { Demanda } \\
\text { Sustituta }\end{array}$ & & \\
\hline 0 & $1.304 .688,00$ & - & - & - & - & - & $(1.304 .688,00)$ \\
\hline 1 & 0 & $30.600,00$ & 22,62 & $2.448,00$ & 164.160 & 16596,00 & $180.634,62$ \\
\hline 2 & 0 & $30.600,00$ & 23,52 & $2.545,68$ & 170.710 & 16596,00 & $187.283,18$ \\
\hline 3 & 0 & $30.600,00$ & 24,46 & $2.647,25$ & 177.521 & 16596,00 & $194.197,02$ \\
\hline 4 & 0 & $30.600,00$ & 25,44 & $2.752,87$ & 184.604 & 16596,00 & $201.386,72$ \\
\hline 5 & 0 & $30.600,00$ & 26,45 & $2.862,71$ & 191.970 & 16596,00 & $208.863,29$ \\
\hline 6 & 0 & $30.600,00$ & 27,51 & $2.976,93$ & 199.630 & 16596,00 & $216.638,18$ \\
\hline 7 & 0 & $30.600,00$ & 28,61 & $3.095,71$ & 207.595 & 16596,00 & $224.723,28$ \\
\hline 8 & 0 & $30.600,00$ & 29,75 & $3.219,23$ & 215.878 & 16596,00 & $233.130,98$ \\
\hline 9 & 0 & $30.600,00$ & 30,93 & $3.347,68$ & 224.492 & 16596,00 & $241.874,15$ \\
\hline 10 & 0 & $30.600,00$ & 32,17 & $3.481,25$ & 233.449 & 16596,00 & $250.966,17$ \\
\hline 11 & 0 & $30.600,00$ & 33,45 & $3.620,16$ & 242.763 & 16596,00 & $260.420,96$ \\
\hline 12 & 0 & $30.600,00$ & 34,79 & $3.764,60$ & 252.450 & 16596,00 & $270.252,99$ \\
\hline 13 & 0 & $30.600,00$ & 36,17 & $3.914,81$ & 262.522 & 16596,00 & $280.477,33$ \\
\hline 14 & 0 & $30.600,00$ & 37,62 & $4.071,01$ & 272.997 & 16596,00 & $291.109,62$ \\
\hline 15 & 0 & $30.600,00$ & 39,12 & $4.233,44$ & 283.890 & 16596,00 & $302.166,13$ \\
\hline 16 & 0 & $30.600,00$ & 40,68 & $4.402,36$ & 295.217 & 16596,00 & $313.663,80$ \\
\hline 17 & 0 & $30.600,00$ & 42,30 & $4.578,01$ & 306.996 & 16596,00 & $325.620,22$ \\
\hline 18 & 0 & $30.600,00$ & 43,99 & $4.760,67$ & 319.245 & 16596,00 & $338.053,71$ \\
\hline 19 & 0 & $30.600,00$ & 45,74 & $4.950,62$ & 331.983 & 16596,00 & $350.983,30$ \\
\hline 20 & 0 & $30.600,00$ & 47,57 & $5.148,15$ & 345.229 & 16596,00 & $364.428,77$ \\
\hline 21 & 0 & $30.600,00$ & 49,47 & $5.353,56$ & 359.004 & 16596,00 & $378.410,72$ \\
\hline 22 & 0 & $30.600,00$ & 51,44 & $5.567,17$ & 373.328 & 16596,00 & $392.950,55$ \\
\hline 23 & 0 & $30.600,00$ & 53,49 & $5.789,30$ & 388.224 & 16596,00 & $408.070,51$ \\
\hline 24 & 0 & $30.600,00$ & 55,63 & $6.020,29$ & 403.714 & 16596,00 & $423.793,77$ \\
\hline 25 & 0 & $30.600,00$ & 57,85 & $6.260,50$ & 419.822 & 16596,00 & $440.144,38$ \\
\hline 26 & 0 & $30.600,00$ & 60,16 & $6.510,30$ & 436.573 & 16596,00 & $457.147,38$ \\
\hline 27 & 0 & $30.600,00$ & 62,56 & $6.770,06$ & 453.992 & 16596,00 & $474.828,80$ \\
\hline 28 & 0 & $30.600,00$ & 65,05 & $7.040,18$ & 472.106 & 16596,00 & $493.215,71$ \\
\hline 29 & 0 & $30.600,00$ & 67,65 & $7.321,09$ & 490.944 & 16596,00 & $512.336,26$ \\
\hline 30 & 0 & $30.600,00$ & 70,35 & $7.613,20$ & 510.532 & 16596,00 & $532.219,71$ \\
\hline 31 & 0 & $30.600,00$ & 73,15 & $7.916,97$ & 530.902 & 16596,00 & $552.896,52$ \\
\hline 32 & 0 & $30.600,00$ & 76,07 & $8.232,85$ & 552.085 & 16596,00 & $574.398,33$ \\
\hline 33 & 0 & $30.600,00$ & 79,11 & $8.561,34$ & 574.114 & 16596,00 & $596.758,07$ \\
\hline 34 & 0 & $30.600,00$ & 82,26 & $8.902,94$ & 597.021 & 16596,00 & $620.009,95$ \\
\hline 35 & 0 & $30.600,00$ & 85,55 & $9.258,17$ & 620.842 & 16596,00 & $644.189,59$ \\
\hline \begin{tabular}{|l|} 
Total \\
\end{tabular} & $1.304 .688,00$ & $1.071 .000,00$ & $1.662,67$ & $179.939,08$ & $12.066 .502,92$ & $580.860,00$ & $12.738 .244,68$ \\
\hline
\end{tabular}

\section{Análisis de Resultados}

La investigación se encuentra sujeta a las corrientes investigativas de carácter cualitativo y cuantitativo, donde se analizarán las estrategias de operación de subestaciones y líneas de subtransmisión en empresas eléctricas públicas.De igual forma, se utilizarán herramientas tecnológicas con el 
objetivo de implementar equipos de automatización, preservando la capacidad humana de los operarios, afin de tener un sistema eléctrico en óptimas condiciones bajo los términos de operatividad de equipos, implementando diseños, presupuestos y poniendo en práctica políticas vigentes en otros países adaptadas a nuestra realidad para circuitos de potencia y circuitos electrónicos de potencia.

Para el siguiente proyecto se ha desarrollado el análisis económico necesariopara verificar si es viable o no realizarlo.Para ello se ha elaborado un flujo de caja (ingresos menos gastos netos) descontando la tasa de interés que se podría haber obtenido menos la inversión inicial dando como resultado un VAN (Valor Actual Neto) de $\$ 11.433 .556,68$, por lo que podemos decir que si se generarán beneficios económicos en el término del proyecto cuya duración es de 35 años. Después, se procede con la fórmula de la TIR cuyo resultado es $17 \%$ y esto comparado con el costo de oportunidad $25 \%$, permitiéndonos concluir que la TIR $<\mathrm{r}$, dando como conclusión que se aprobara el proyecto.

El análisis de factibilidad económica se realizará desde el punto de vista de la cogeneración" inyección de voltaje por energía renovable”. Esta energía es suministrada por el Ingenio San Carlos. El valor de generación limpia soporta 10.2 MW por un período de 15 minutos, tiempo que tarda la desconexión de las empresas antes detalladas por motivo de cambio de alimentación de una línea de subtransmisión a otra. En este caso al alimentar la subestación desde la línea Milagro 4 el valor que la empresa eléctrica pierde por desconexión de la carga es de \$367.200.00. (Avila-PratsI, AlesancoGarcíaI \& Veliz-AlonsoII, 2011). Estos proyectos aplicados mediante energía renovable deben evaluar impactos ambientales, energéticos, de acuerdo a incidencias demográficas, con el objetivo de implementar el recurso apropiado.

\section{Conclusiones}

La investigación cuantitativa hace referencia al análisis económico financiero, a través de magnitudes que pueden ser tratadas mediante el uso de herramientas estadísticas en niveles de recuperación de carga, implementación de equipos automatizados de transferencia así como también equipos de potencia en subestación y análisis de índices de calidad FMIK y TTIK . La investigación cualitativa se enfoca en la recopilación de información de políticas antiguas analizadas en términos de automatización, es por ello que concluimos que siguiendo las recomendaciones del sistema de potencia a implementar garantizamos un sistema de potencia confiable, con calidad de servicio en lo que respecta a parámetros eléctricos. Nos responsabilizamos igualmente por la integridad personal del operario pues las lecturas de los parámetros eléctricos pueden ser monitoreadas vía remota por el centro de 
operaciones de CNLE EP UN MLG. Este monitoreo es en tiempo real, en el cual se pueden observar los niveles de tensión, corriente, potencia aparente, reactiva y activa.

Con el monitoreo al sistema SCADA se podrán identificar los parámetros afectados bajo cualquier contingencia. Este está destinado para el transformador de poder, el equipo de potencia más importante y considerado como el corazón de la subestación. Como monitoreo al transformador de potencia tenemos monitoreo a la temperatura del aceite, bobinado y cromatografía de gases donde se puede observar el estado del papel y la incidencia de chisporroteo en el núcleo.

Todo lo anterior garantiza la confiablidad de un sistema eléctrico de potencia y gran calidad de servicio mediante la realización de un mantenimiento adecuado y la existencia de un área de trabajo con menos riesgo eminente de descargas eléctricas por contacto El proyecto es entonces rentable dentro de los primeros años con una tasa de rentabilidad alta, justificando la inversión y la toma de decisiones para implementar al solución del problema de la SUBESTACION MARECELINO MARIDUEÑA.

El sistema eléctrico de media tensión tiene que ser automatizado para disminuir los tiempos de respuesta conmotores con un voltaje a 125 VDC, permitiendo tener mayor torque en el accionamiento mecánico.

El SCADA implementado en las empresas eléctricas del estado ecuatoriano es utilizado para supervisión y monitoreo de unidades de guardia, mediante un sistema georeferenciado para contingencias que afecten la fluidez del servicio eléctrico.

El SCADA deberá ser integrado a una plataforma virtual que permita visualizar en tiempo real el estado de la subestación, los equipos de potencia y el transformador de poder, obteniendo así un esquema de contingencia para posibles fallas. Gracias a esto se podría identificarla posible falla bajo mediante simulaciones, disminuyendo así los tiempos de interrupción; esto conlleva al mejoramiento de indicadores de calidad de servicio FMIK y TTIK.

Las fuentes de energía renovable se deben integrar a la matriz energética, ya que con su estudio, análisis de factibilidad e implementación, reduciremos la dependencia del uso de fuentes de energía de origen fósil.

\section{Recomendaciones}

Es necesaria la implementación del sistema de eliminación de armónicos ya que garantiza la eliminación de daños en equipos electrónicos por transferencia de carga y fluctuación de voltaje de las generadoras de energía eléctrica.

El diseño estratégico de operación garantiza la confiablidad del sistema eléctrico de potencia para consumidores de tipo residencial, comercial e 
industrial, permitiendo obtener niveles óptimos de voltaje y un sistema confiable.

El reemplazo de equipos seguido de la puesta en práctica de la protección para equipos electrónicos, garantiza la continuidad del servicio ya que aunado a un buen mantenimiento preventivo, correctivo y predictivo se garantiza la protección humana y el tiempo de vida de los equipos de potencia.

\section{References:}

1. Acero, P. (2018). DIRECCION ESTRATEGICA. Ecoe Ediciones, 2018.

2. AGENCIA DE REGULACION Y CONTROL DE ELECTRICIDAD (ENERO de 2018). CNEL EP PLIEGO TARIFARIO. Obtenido de https://www.cnelep.gob.ec/pliego-tarifario-2/

3. Aldo Gary Arriaga Mass (1994). DISEÑO DE UN SISTEMA DE MONITOREO DE PARÁMETROS ELÉCTRICOS. DISEÑO DE UN SISTEMA DE MONITOREO DE PARÁMETROS ELÉCTRICOS. Santiago de Chile, Chile.

4. Altomonte, H., Coviello, M., \& FLutz, W. (2003). Energias renovables y eficiencia energética en América Latina y el Caribe Restricciones y Perspectivas. Scielo, 71.

5. ARCONEL (2018). Numero de Abonados Cobertura CNEL EP UN MILAGRO. Milagro.

6. ARIZA, D.A. (2017). EFECTIVIDAD DE LA GESTION DE LOS PROYECTOS: UNA PERSPECTIVA CONSTRUCTIVISTA. OBRAS Y PROYECTOS, 75-85.

7. Avella, H. \& Andrés, S. (2016). Diagnóstico de las subestaciones con sistema de automatización y control propiedad de la EBSA. Scielo, 73.

8. Avila-PratsI, D., Alesanco-GarcíaI, R., \& Veliz-AlonsoII, J. (2011). Sistemas híbridos con base en las energías renovables para el suministro de energía a plantas desaladoras. Scielo, 30.

9. Borges, C. G. \& Cera, A. S. (2010). Dimensionado mediante simulación de sistemas de energía solar fotovoltaica aplicados a la electrificación rural. Scielo, 9.

10. ELECTRICIDAD, C. N. (2014). RENDICION DE CUENTA . Guayaquil.

11. Energético, U. D. (2015). Integración de las Energías Renovables en Colombia. Obtenido de http://www.upme.gov.co/Estudios Integracion_Energias_Renovables/INTEGRACION_ENERGIAS_R ENOVANLES_WEB.pdf

12. Garcia Jhon - Rendon, G. A. (2018). Redes inteligentes y mecanismo de respuesta de la demanda: el caso del sector eléctrico colombiano. Universidad EAFIT - School of Economics and Finance - Center for 
Research in Economic \& Finance (CIEF), Center for Research in Economics and Finance (CIEF), Working Papers, No. 18-20.

13. González-Ávila, M. E., BeltrÁn-Morales, L. F., Troyo-Diéguez, E., \& Ortega-Rubio, A. (2004). POTENCIAL DE APROVECHAMIENTO DE LA ENERGÍA EÓLICA PARA LA GENERACIÓN DE ENERGÍA ELÉCTRICA EN ZONAS RURALES DE MÉXICO. Scielo, 10.

14. Guachún Villalta, L. M. (2018). Estudio de factibilidad de un sistema de energía renovable aplicado a un nodo de servicio de telecomunicaciones. Cuenca, Ecuador.

15. GUTIERREZ LASCURAIN ISABELA (2012). DIAGNOSTICO Y PROPUESTA DE MEJORA DE CALIDAD EN EL SERVICIO DE UNA EMPRESA DE UNIDADES DE ENERGIA ELECTRICA. MEJORA DE CALIDAD EN EL SERVICIO DE UNA EMPRESA DE UNIDADES DE ENERGIA ELECTRICA. MEXICO, MEXICO.

16. Hurtado Avélla \& Santiago Andrés (2016). Diagnóstico de las subestaciones con sistema de automatización y control propiedad de la EBSA. Diagnóstico de las subestaciones con sistema de automatización y control propiedad de la EBSA. Tunya, Colombia: Universidad Pedagógica y Tecnológica de Colombia.

17. José Antonio Pérez Fernández de Velasco (2010). Gestión por procesos. España: ESIC EDITORIAL.

18. Lourdes, M., Hanoi, S., \& Eduardo, O. (2005). IMPACTO DE ALGUNAS TECNOLOGÍAS EN EL DESARROLLO DE LOS SISTEMAS SCADA. Scielo, 15.

19. Mendoza Segura, J. S. (5 de 12 de 2017). DISEÑO DE UN SISTEMA DE MONITOREO DE PARÁMETROS ELÉCTRICOS. DISEÑO DE UN SISTEMA DE MONITOREO DE PARÁMETROS ELÉCTRICOS. LIma, Peru.

20. Mendoza Segura \& Joel Shakespeare (5 de 12 de 2017). DISEÑO DE UN SISTEMA DE MONITOREO DE PARÁMETROS ELÉCTRICOS. DISEÑO DE UN SISTEMA DE MONITOREO DE PARÁMETROS ELÉCTRICOS. Lima, Peru.

21. Mohali, G. \& Ascanio, P. (. (2010). Confiabilidad estructural y políticas de mantenimiento a la subestación eléctrica Yaritagua $115 / 13,8 \mathrm{kV}$. Scielo, 8.

22. Mundial, E. d. (2017). Origen de la Gestion de Proyectos.

23. Nauí, C. Y. (2015). Twenergy. Obtenido de Twenergy: https://twenergy.com/co/a/que-son-las-subestaciones-electricas-ypara-que-sirven-1759 
24. Pérez, B. E., Pérez, E. I., Rada, J. L., Lima, J. R., Rodríguez, B. S., \& Díaz, H. M. (2011). Sistema para el diseño de Subestaciones Electricas para Distriubución. Tendencia tecnológica, 8.

25. Pineda Bernabel \& Ronald David (Noviembre de 2017). Ingeniería de valor aplicada a la administración de proyectos: saneamiento de sistemas operativos - proyecto modernización refinería Talara. Cajamarca,

Peru: http://repositorio.upagu.edu.pe/handle/UPAGU/401.

26. Planificación, S. N. \& Desarrollo, y. (2013). Plan Nacional del Buen Vivir del Ecuador. Obtenido de Plan Nacional del Buen Vivir del Ecuador: http://www.buenvivir.gob.ec/objetivos-nacionales-para-elbuen-vivir

27. Quispe Flores \& Juvenal Antonio (04 de 09 de 2018). Protección y coordinación del sistema eléctrico rural de media tensión en $22,9 \mathrm{KV}$, para mejorar la calidad del servicio de energía eléctrica del Distrito de Ichuña - Provincia General Sánchez Cerro - Región Moquegua, Año 2016. MOQUEGUA, PERU.

28. Romero Romano \& Carlos Osvaldo (2018). Estrategia para el fortalecimiento de la producción y comercialización de.

29. Secretaría Nacional de Planificación y Desarrollo, S. (11 de 10 de 2017). El Plan Nacional de Desarrollo (denominado "Plan Nacional de Desarrollo 2017-2021. Obtenido de www.planificacion.gob.ec/wpcontent/.../PNBV-26-OCT-FINAL_0K.compressed1.pdf

30. Spiegeler, C. \& Cifuentes, J. I. (s.f.). DEFINICION E INFORMACION DE ENERGIAS RENOVABLES. http://www.repositorio.usac.edu.gt/4455/1/DEFINICION\%20E\%20I NFORMACION\%20DE\%20ENERGIAS\%20RENOVABLES.pdf, 7.

31. Valarezo Vera \& Genith Maria (2018). Análisis de la factibilidad de un plan de desarrollo social urbanístico de viviendas de interés social proyecto "La pierina" en el cantón de Chone. Guayaquil, Ecuador: Universidad de Guayaquil Facultad de Ciencias Administrativas.

32. Viteri Toquica Diego1, G. B. (10 de 12 de 2016). AnalisisdeConfiabilidadenSubestaciones

ElectricasTipoManiobraImplementandoel

TransformadordeTensi'onconN'ucleodePotencia. Bogota, Colombia.

33. WENDY PAOLA PERUGACHI FLORES (3 de 01 de 2018). DISEÑO DE UN MODELO DE GESTIÓN POR PROCESOS PARA. DISEÑO DE UN MODELO DE GESTIÓN POR PROCESOS PARA. Quito, Ecuador. 